\title{
Vetting Neutral Nitrogen Vacancies
}

\author{
New experiments characterize the excitation levels of electrically neutral \\ nitrogen-vacancy centers, information needed for quantum information \\ applications.
}

\section{By Michael Schirber}

N itrogen-vacancy centers are small defects in diamond crystals, which can perform many functions in quantum information and sensing technologies (see Q\&A: Defects Wanted; Apply Here). Negatively charged (NV ${ }^{-}$) centers-those with one extra electron-have proven to be the most useful, but the defects also come in a less-studied neutral state $\left(\mathrm{NV}^{0}\right)$. Now, Simon Baier from Delft University of Technology in the Netherlands and his colleagues have performed a series of optical spectroscopy experiments that reveal the excitation levels in $\mathrm{NV}^{0}$ centers, knowledge that could improve the applicability of nitrogen-vacancy centers [1].

Like an atom, nitrogen-vacancy centers have several bound electrons, which can reside in one of many orbitals. The $\mathrm{NV}^{-}$center-with six electrons-is prized for its long-lived spin states that can store quantum information. However, under laser excitation an $\mathrm{NV}^{-}$center can spontaneously lose an electron and switch to $\mathrm{NV}^{0}$, resulting in a loss of signal and the

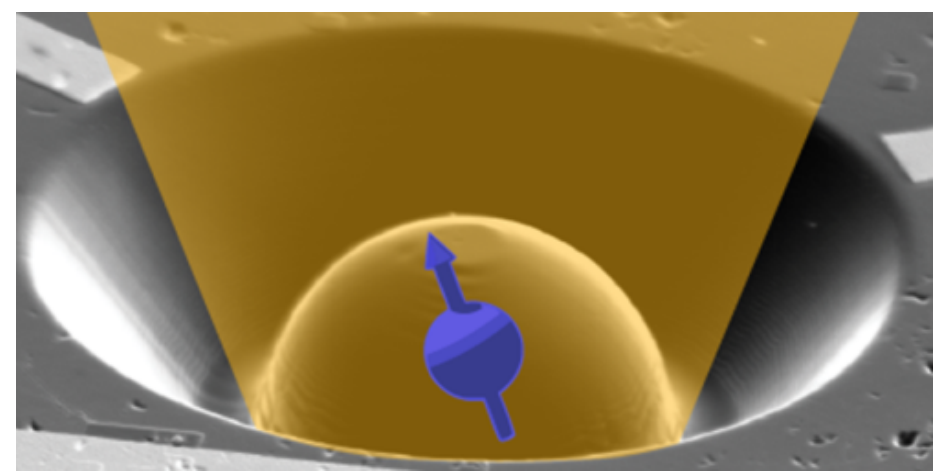

Credit: QuTech/Delft University of Technology decoherence of nearby qubits. Those problems could be mitigated if the $\mathrm{NV}^{0}$ center's spin properties were better understood.

$\mathrm{NV}^{0}$ centers rapidly undergo transitions, making it difficult to identify the initial and final states of a given transition. Baier and colleagues overcome this problem by developing a technique that can carefully place a single nitrogen-vacancy center in a well-defined state. By monitoring the light emission from this targeted center, they showed that they could clearly identify transitions involving orbital-state changes from those involving spin-state changes. They also measured how the spin states evolve, both in the dark and under laser illumination. They then used this information to demonstrate a low-error (high-fidelity) readout technique of the $\mathrm{NV}^{0}$ spin state that could be used in future qubit applications.

Michael Schirber is a Corresponding Editor for Physics based in Lyon, France.

\section{REFERENCES}

1. S. Baier et al., "Orbital and spin dynamics of single neutrally-charged nitrogen-vacancy centers in diamond," Phys. Rev. Lett. 125, 193601 (2020). 